\title{
ON NEARLY COMMUTATIVE NODAL ALGEBRAS IN CHARACTERISTIC ZERO
}

\author{
MICHAEL RICH
}

\begin{abstract}
In this paper we consider algebras satisfying the identity (I) $x(x y)+(y x) x=2(x y) x$ and show that there are no nodal algebras of this type over any field $F$ of characteristic zero. The proof is obtained by first showing that if $x$ is an element of a finitedimensional algebra satisf ying (I) over a field of characteristic zero then the operator $L(x)-R(x)$ is nilpotent.
\end{abstract}

A finite-dimensional power-associative algebra $A$ with identity 1 over a field $F$ is called a nodal algebra [7] if every $x$ in $A$ is of the form $x=\alpha 1+n$ where $\alpha$ is in $F$ and $n$ is nilpotent, and the set $N$ of nilpotent elements of $A$ does not form a subalgebra of $A$. Albert [1] has proved that there are no commutative nodal algebras over any field $F$ of characteristic zero by showing that $N$ forms a subalgebra. There do exist, however, examples of nodal algebras over fields of characteristic zero [2].

Algebras satisfying (I) have been studied by Kosier [5], Witthoft [8] and the author [6]. It should be noted that in linearized form (I) reduces to

$$
x(z y)+z(x y)+(y x) z+(y z) x=2(x y) z+2(z y) x
$$

and in operator form (I) is just

$$
L(x)^{2}+R(x)^{2}=2 L(x) R(x)
$$

or

$$
L(x)(L(x)-R(x))=(L(x)-R(x)) R(x)
$$

where $L(x)(R(x))$ is the operator which acts as follows: $y L(x)$ $=x y(y R(x)=y x)$.

In a commutative algebra $L(x)-R(x)=0$. For algebras satisfying (I) we have the following.

Theorem 1. Let $A$ be a finite-dimensional algebra satisfying (I) over a field $F$ of characteristic zero. Then for any element $x$ the operator $L(x)-R(x)$ is nilpotent.

Received by the editors June 27, 1969 and, in revised form, September 15, 1969. AMS Subject Classifications. Primary 1720.

Key Words and Phrases. Nodal algebra, power-associative, characteristic zero, nilpotent, finite-dimensional.

1 The author is indebted to the referee for his suggestions in shortening the proof of Theorem 1. 
Proof. It is known $[4$, p. 43] that a transformation $T$ on a finitedimensional vector space $V$ over a field of characteristic zero is nilpotent if all of its powers have trace zero; i.e., $\operatorname{tr} T^{n}=0(n=1,2, \cdots)$. We show that $T=(L(x)-R(x))^{2}$ is nilpotent. Consider $\operatorname{tr}\left[(L(x)-R(x))^{m}\right]$ for $m \geqq 2$. Clearly,

$$
\begin{aligned}
\operatorname{tr}\left[(L(x)-R(x))^{m}\right]= & \operatorname{tr}\left[L(x)(L(x)-R(x))(L(x)-R(x))^{m-2}\right] \\
& -\operatorname{tr}\left[R(x)(L(x)-R(x))^{m-2}(L(x)-R(x))\right]
\end{aligned}
$$

now, by (3), $L(x)(L(x)-R(x))=(L(x)-R(x)) R(x)$ and by the commutativity of the trace, $\operatorname{tr}\left[R(x)(L(x)-R(x))^{m-2}(L(x)-R(x))\right]=$ $\operatorname{tr}\left[(L(x)-R(x)) R(x)\left(L(x)-R(x)^{m-2}\right)\right]$. Therefore,

$$
\begin{aligned}
\operatorname{tr}\left[(L(x)-R(x))^{m}\right]= & \operatorname{tr}\left[(L(x)-R(x)) R(x)(L(x)-R(x))^{m-2}\right] \\
& -\operatorname{tr}\left[(L(x)-R(x)) R(x)(L(x)-R(x))^{m-2}\right] \\
= & 0 .
\end{aligned}
$$

Therefore, $(L(x)-R(x))^{2}$ is nilpotent.

Corollary. For $A$ as above, $\operatorname{tr} L(x)=\operatorname{tr} R(x)$ for any element $x$.

It should be noted that if $A$ is flexible then Theorem 1 is trivial since then (2) is just $(L(x)-R(x))^{2}=0$.

THEOREM 2. There do not exist any nodal algebras satisfying $x(x y)$ $+(y x) x=2(x y) x$ over any field $F$ of characteristic zero.

Proof. Gerstenhaber [3, p. 29] has shown that in a commutative power-associative algebra over a field $F$ of characteristic zero, the assumption that an element $n$ is nilpotent implies that $R(n)$ is nilpotent. If an algebra $A$ is not commutative this result can be applied to $A^{+}$where $A^{+}$is the same vector space as $A$ but multiplication in $A^{+}$is given by: $x \cdot y=\frac{1}{2}(x y+y x), x y$ the multiplication in $A$. Thus, if $a$ is nilpotent in $A$ then $R(a)^{+}=\frac{1}{2}(R(a)+L(a))$ is nilpotent and $\operatorname{tr} R(a)+\operatorname{tr} L(a)=0$.

Now let $A$ be a finite-dimensional power-associative algebra satisfying (I) over a field $F$ of characteristic zero, every element $a$ of $A$ being of the form $a=\alpha 1+n$ with $n$ nilpotent. We show that the set $N$ of nilpotent elements of $A$ forms a subalgebra. As in [6, Theorem 1] write (1) in terms of operators to get,

(4) $R(y) L(x)+R(x y)+L(y x)+L(y) R(x)=2 L(x y)+2 R(y) R(x)$. 
If we interchange $x$ and $y$ in (4) and subtract the result from (4) we have: $[L(y), R(x)]+[R(y), L(x)]+R([x, y])+L([y, x])=2 L([x, y])$ $+2[R(y), R(x)]$ (as usual $[A, B]$ denotes $A B-B A$ ) which gives rise to:

$$
\operatorname{tr}(R[x, y])+\operatorname{tr} L([y, x])=2 \operatorname{tr} L([x, y]) .
$$

Let $[x, y]=-[y, x]=\alpha 1+n$ with $\alpha$ in $F$ and $n$ nilpotent. By [3] $\operatorname{tr} L(n)+\operatorname{tr} R(n)=0$. By the corollary $\operatorname{tr} L(n)=\operatorname{tr} R(n)$. Therefore $\operatorname{tr} L(n)=\operatorname{tr} R(n)=0$. Thus (5) reduces to: $\operatorname{tr} R(\alpha 1)-\operatorname{tr} L(\alpha 1)$ $=2 \operatorname{tr} L(\alpha 1)$ or $2 \alpha \operatorname{dim} A=0$. Therefore $\alpha=0$. In particular, if $x$ and $y$ are in $N$ then $[x, y]$ is in $N$. But by [1] $x y+y x$ is in $N$. Therefore $x y$ and $y x$ are in $N, N$ is a subalgebra and $A$ is not a nodal algebra.

\section{REFERENCES}

1. A. A. Albert, $A$ theory of power-associative commutative algebras, Trans. Amer. Math. Soc. 69 (1950), 503-527. MR 12, 475.

2. T. A. Anderson, $A$ note on derivations of commutative algebras, Proc. Amer. Math. Soc. 17 (1966), 1199-1202. MR 34 \#309.

3. M. Gerstenhaber, On nilalgebras and linear varieties of nilpotent matrices. II, Duke Math. J. 27 (1960), 21-32. MR 22 \#742.

4. N. Jacobson, Lie algebras, Interscience Tracts in Pure and Appl. Math. no. 10, Interscience, New York, 1962. MR 26 \#1345.

5. F. Kosier, On a class of nonflexible algebras, Trans. Amer. Math. Soc. 102 (1962), 299-318. MR 24 \#A3187.

6. M. Rich, On a class of nodal algebras, Pacific J. Math. 32 (1970).

7. R. D. Schafer, On non-commutative Jordan algebras, Proc. Amer. Math. Soc. 9 (1958), 110-117. MR 21 \#2677.

8. W. G. Witthoft, $A$ class of nilstable algebras, Trans. Amer. Math. Soc. 111 (1964), 413-422. MR 28 \#4015.

Temple University 